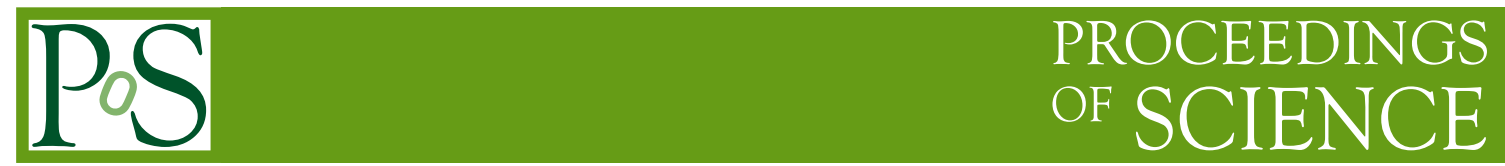

\title{
Strangeness production on the neutron
}

\section{Colin Wilkin*}

Physics and Astronomy Department, UCL, Gower Street, London WC1E 6BT, United Kingdom

E-mail: cw@hep.ucl.ac.uk

The production of hyperons in neutron-proton collisions near threshold is surveyed. The comparison of COSY-ANKE experimental data on inclusive $K^{+}$production in $p d$ and $p p$ collisions shows that $\Lambda$ production is about as strong in the $p n$ channel as in $p p$. This is in agreement with a theoretical prediction. Exclusive measurements, involving the detection of either $K^{0}$ or $K^{+}$ mesons, have been also carried out at COSY-TOF and COSY-ANKE, respectively. The current status of these two experiments is discussed.

8th International Conference on Nuclear Physics at Storage Rings-Stori11,

October 9-14, 2011

INFN Laboratori Nazionali di Frascati, Italy

${ }^{*}$ Speaker. 


\section{Introduction}

There are three strangeness-conserving $K^{+}$production channels open in $p p$ collisions close to threshold. The most widely investigated is $p p \rightarrow K^{+} p \Lambda$, for which data on the total cross sections, Dalitz plots, and angular spectra exist. Total cross sections and angular distributions are also available for $p p \rightarrow K^{+} p \Sigma^{0}$. The energy dependence of the total cross sections is determined mainly by phase space, modified in the $\Lambda$ case by a strong $\Lambda p$ final state interaction (FSI) and the possible effects from $N^{*}$ resonances. In addition to these two channels, recent data suggest that the total cross section $\sigma\left(p p \rightarrow p n \Sigma^{+}\right) \approx 0.7 \sigma\left(p p \rightarrow K^{+} p \Sigma^{0}\right)$. A detailed bibliography on the $p p$ reactions is to be found in Ref. [1] but, in contrast, almost nothing is known about hyperon production in proton-neutron collisions.

It is important to determine the ratio of $K^{+}$in $p n$ and $p p$ collisions, $R=\sigma_{p n}^{K^{+}} / \sigma_{p p}^{K^{+}}$, for many reasons but its value is a crucial ingredient for:

Modelling of $K^{+}$production in proton-nucleus and nucleus-nucleus collisions, and Modelling of $\mathrm{K}^{+}$production in nucleon-nucleon collisions.

As reported in Ref. [1], there have been several indirect attempts to estimate $K^{+}$production in $p n$ reactions from experimental data:

- $3 \mathrm{GeV} p A$ collisions $\Rightarrow R=5 \pm 7.5$,

- $2.1 \mathrm{GeV} / A$ protons and deuterons incident on $\mathrm{NaF} \Rightarrow R<1$,

- $2.5 \mathrm{GeV}$ Comparison of $p p$ and $p \mathrm{C}$ collisions $\Rightarrow R \approx 1$,

- $2.02 \mathrm{GeV}$ proton-deuteron collisions $\Rightarrow R \approx 3$ ( but very model-dependent).

Since there is nothing terribly convincing here, let us turn to theory.

Most of the calculations of $N N \rightarrow K^{+} N \Lambda$ in the literature are some variety of a one-mesonexchange model, which can be dressed with initial and final state interactions. However, there is no consensus as to which meson exchanges are important!

- COSY-TOF data show the strong influence of the $N^{*}(1650)$ isobar decaying into $K^{+} \Lambda$, and this can only come about through non-strange exchange [2].

- The spin-transfer parameter $D_{N N}$ from the proton to the hyperon in $\vec{p} p \rightarrow K^{+} p \vec{\Lambda}$ has been interpreted as favouring strangeness exchange [3].

The $N^{*}$ evidence is much stronger but, even if one keeps only non-strange exchange, the results depend enormously on the relative strengths of the $\pi$ versus $\rho$ contributions.

Near-threshold $\eta$ production in $N N$ collisions is driven mainly by the $S_{11}(1535)$, whereas nearthreshold $K^{+}$production is driven mainly by the $S_{11}(1650)$ isobar so that there is a lot of similarity between the two productions. Now $\eta$ production in $p n$ collisions is about 6.5 times stronger than in $p p$, i.e., the isospin $I=0$ cross section is about TWELVE times stronger than that of $I=1$ [4].

Theoretical modelling suggests that, if one keeps only $\pi$ and $\rho$ exchanges, there is destructive interference between them for $p p \rightarrow p p \eta$ but constructive for $p n \rightarrow p n \eta$. A quantitative description of the available data can be achieved if one estimates the $\rho$ coupling to the $S_{11}(1535)$ using 
Vector Meson Dominance and photoproduction data. This gives the ratio of the two production amplitudes $x=D_{\pi} / B_{\rho}=0.7$ [5].

In order to estimate the equivalent ratio for $K^{+}$production, we scale the value of $x$ by the ratio of the $K \Lambda$ and $\eta N$ production amplitudes in $\pi^{-} p$ and $\gamma p$ collisions. If the two productions are each driven by a single $S_{11}$ isobar, then $x$ should be dominantly real, though its sign is undetermined. The two possible values are then $x= \pm(0.9 \pm 0.2)$, depending whether one has constructive or destructive $\pi / \rho$ interference. In the theoretical work, only the positive solution for $x$ was considered and this led to a $p n / p p$ ratio of $R \approx 7$. On the other hand, the negative solution for $x$ would give $R=0.5 \pm 0.2$. It is clear that, without fixing the signs of the coupling of the $\pi$ and $\rho$ to the two $S_{11}$ isobars, one will always end up with two possible values of $R$ [5].

\section{Inclusive $K^{+}$production}

The first indication that $\eta$ production was much stronger in the $p n$ channel than in $p p$ came from a PINOT measurement of inclusive $\eta$ production in $p d$ and $p d$ collisions [6]. It was suggested that the same methodology could be employed for inclusive $K^{+}$production and this has been used at COSY-ANKE [1].

The experiments were carried out at the Cooler-Synchrotron COSY-Jülich at 1.83, 1.92, 2.02, and $2.65 \mathrm{GeV}$ using unpolarized proton beams incident on hydrogen and deuterium cluster-jet targets. The resulting reaction products were detected in the ANKE magnetic spectrometer, which is situated in a chicane inside the COSY ring. The spectrometer uses three dipole magnets. D1 and D3 divert the circulating beam onto the ANKE target and back into the COSY ring, respectively, while $D 2$ is the analysing magnet.

The crucial element in the approach is the positive detector of fifteen range telescopes placed in the focal plane of $D 2$. These study the products of the $K^{+}$delayed decay. The time measurements allow a clear $K^{+}$identification even when the background from protons and $\pi^{+}$is $10^{6}$ times higher. Inclusive kaon production would only require the use of these range telescopes and this would be completely equivalent to the PINOT $\eta$ experiment [6]. However, in contrast to the $\eta$ measurement, this was an internal measurement and the forward detector was needed to measure the fast proton from elastic $p p$ or $p d$ scattering (or breakup) in order to determine the luminosity. In the $p d$ case the luminosity could also be fixed by measuring the recoil deuteron from elastic $p d$ scattering in a silicon tracking telescope. The typical luminosity uncertainty was $\approx 10-15 \%$. The resulting laboratory double-differential cross sections determined for $K^{+}$production in $p p$ and $p d$ collisions, averaged over production angles $\theta_{K}<4^{\circ}$, are shown in Fig. 1

Also shown in the figure are the predictions in a three-channel model where, in the $p p$ case, we only consider contributions from the $p p \rightarrow K^{+} p \Lambda, p p \rightarrow K^{+} p \Sigma^{0}$, and $p p \rightarrow K^{+} n \Sigma^{+}$reactions. Here it is assumed that $\Sigma$ production follows phase space but, for the $\Lambda$, effects from the $\Lambda p$ FSI and $N^{*}(1650)$ have been included. The normalisations were fixed in terms of the measured total cross sections. As can be seen from the figure, smearing over the Fermi motion in the deuteron is unimportant. This model describes reasonably well the ANKE data at the three lower energies and gives confidence that these small angle data are typical of the behaviour over the full angular domain. Of course, this model fails completely at the highest energy, when many more than three channels are open. 


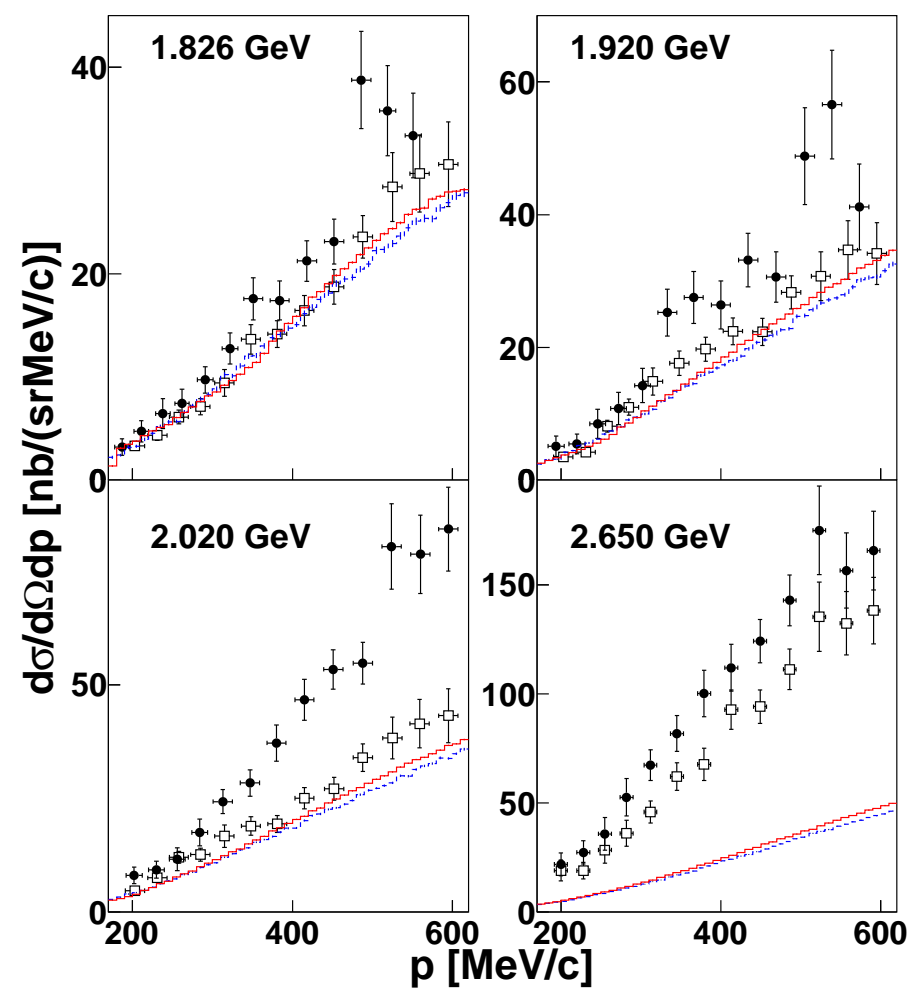

Figure 1: Momentum spectra of $K^{+}$produced in $p p$ (open circles) and $p d$ (closed circles) collisions at 1.826, $1.920,2.020$, and $2.650 \mathrm{GeV}$. Only statistical errors are shown. The red lines represent the description of the $p p$ data within the three-channel model whereas for the blue dashed ones the predictions have been smeared over the deuteron Fermi momentum and a correction made for the shadowing in the deuteron.

Averaging the ratio of cross sections shown in Fig. 1 over the whole $K^{+}$momentum range leads to the mean values shown in Fig. 2 for the four beam energies. The black solid line marked $R=1$ represents the ratio of Fermi-smeared $p p \rightarrow K^{+} p \Lambda$ plus $p p \rightarrow K^{+} N \Sigma$ total cross sections divided by the free hydrogen data. The red dashed line represents the same ratio but evaluated from the differential spectra. The $R=1.5$ lines are simply 1.5 times these values.

The weighted average of the data at the three lowest energies gives $\sigma_{p d}^{K^{+}} / \sigma_{p p}^{K^{+}}=1.4 \pm 0.2$ which, after taking the shadowing into account, means that the ratio for $K^{+}$production in $p n$ and $p p$ collisions

$$
\sigma_{p n}^{K^{+}} / \sigma_{p p}^{K^{+}}=0.5 \pm 0.2
$$

This coincides with the theoretical solution where $\pi / \rho$ interference is destructive for $p n$, which is the opposite to that found for $\eta$ production [5]. Although the ratio seems to depend only weakly upon the beam energy, it should be noted that many different hyperons (and even $\mathrm{K}^{+} K^{-}$pairs) can be produced at $2.65 \mathrm{GeV}$ and this can change the physics significantly.

At low energies, where only $\Lambda$ production is possible, the $0.5 \pm 0.2$ is comfortably above the isospin lower bound of $R>0.25$ [7]. The result translates to

$$
\sigma^{I=0}(N N \rightarrow K N \Lambda) / \sigma^{I=1}(N N \rightarrow K N \Lambda)=1.0 \pm 0.8 .
$$




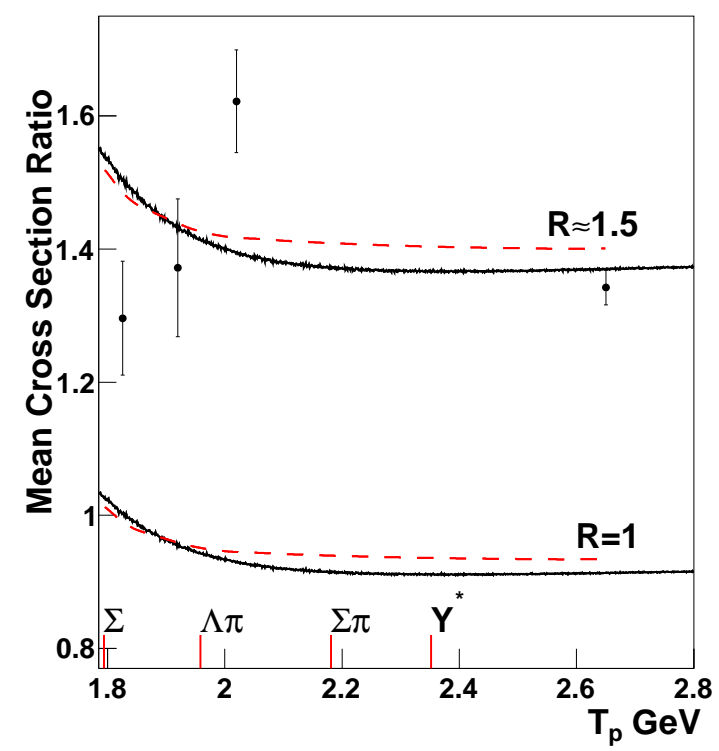

Figure 2: Ratio of $\mathrm{K}^{+}$production cross sections on deuterium to hydrogen, averaged over the kaon momentum range shown in Fig. 1. Errors due to the overall normalisations are not included in the error bars. The lines are described in the text.

It should, of course, be noted that, for a neutron target, half of the signal would be associated with $K^{0}$ production.

\section{Exclusive hyperon production experiments}

The Time-of-Flight spectrometer at COSY, COSY-TOF, has great advantages for the measurement of the production of strange particles close to threshold [8]. Although it has no magnetic field and relies for the identification of an event on the use of kinematic relations involving geometry and timing, the trump card is the fact that strange particles typically decay within the apparatus and so the number of tracks suddenly jumps. Thus, in a reaction such as $p d \rightarrow p_{\mathrm{sp}} K^{0} p \Lambda$, one initially sees just after the target only the fast proton, the "spectator" proton $p_{\text {sp }}$ being undetectable. However, after the $\Lambda \rightarrow p \pi^{-}$and $K^{0} \rightarrow \pi^{+} \pi^{-}$decays, five tracks are seen, which is an excellent signal for a good event. After applying kinematic fitting, the momentum of the unobserved "spectator" proton is reconstructed [9]. COSY-TOF has a very large geometric acceptance but there are clearly problems when the relevant cross section is but a small fraction of the total.

However, the main difficulty is identifying the two decay vertices from the four tracks with their measurement errors - the combinatorial background. One first checks that one pair is consistent with the $\Lambda$ and then see if the other could fit the $K_{s}^{0}$. In practice, this combinatorial background is much more of a problem than the physical background. Use is now being made of the timing information from the start to the stop counters, though this might introduce a bias that has to be understood [10]. 
Of the 85 million events recorded in 32 hours of measurement time, $60 \%$ had been analysed by March 2011. In total, between 300 and 600 reconstructed $K^{0} p \Lambda$ events are expected, depending upon selection criteria. The initial (VERY preliminary) estimate of the total cross section at a beam energy of $2.261 \mathrm{GeV}$ is

$$
\sigma\left(p d \rightarrow p_{\mathrm{sp}} K^{0} p \Lambda\right)=(5.0 \pm 0.5 \pm 1.0) \mu \mathrm{b}
$$

No attempt has yet been made to bin these data in terms of the excess energy but, taken at face value, they would suggest that

$R=\sigma\left(p n \rightarrow K^{0} p \Lambda\right) / \sigma\left(p p \rightarrow K^{+} p \Lambda\right)=\sigma\left(p n \rightarrow K^{+} n \Lambda\right) / \sigma\left(p p \rightarrow K^{+} p \Lambda\right)=0.20 \pm 0.02_{\text {stat }} \pm 0.04_{\text {sys }}$,

which risks falling below the isospin lower bound of 0.25 . It is also low compared to the ANKE result [1], obtained closer to threshold, and bubble chamber data at higher energies [11].

The $p d \rightarrow p_{\mathrm{sp}} K^{0} p \Lambda$ reaction seems to be at the limit of what the original COSY-TOF facility could handle, though the apparatus has been significantly improved since these data were taken. There is only one directly visible prompt track (the fast proton) and only few track points from the decay particles. In addition, the separation of the decays of the $\Lambda$ and the $K_{s}^{0}$ needs "some tricks" and the use of the timing information. The treatment of the timing methodology will be checked in the analysis of a second data set taken at $2.157 \mathrm{GeV}[10]$.

In contrast to the COSY-TOF approach, at ANKE the spectator proton is measured directly and unambiguously in one of the silicon tracking telescopes placed in the vacuum of the target chamber [12]. Since the backgrounds for $K^{+}$detection is so low in the range telescopes, this means that below the $\Sigma$ thresholds all $p_{\mathrm{sp}} K^{+}$coincidence must correspond to the $p_{\mathrm{sp}} K^{+} n \Lambda$ final state. Furthermore, the angle and energy of the spectator proton fixes the cm energy in the $p n \rightarrow K^{+} n \Lambda$ reaction.

The major problem of this method comes from the STT design, which consists of three doublesided silicon strip detectors [13]. In order to determine the angle well, the proton has to penetrate through the first layer and pass into the second. This requires that the proton to have an energy above $2.5 \mathrm{MeV}$, i.e., a momentum above $68 \mathrm{MeV} / c$. This is high for a deuteron Fermi momentum and about half the events are lost because of this cut!

Luminosity will be determined to better than $\approx 5 \%$ by measuring the frequency change in the machine due to the energy loss in the beam-target interactions [14]. The value of luminosity will be checked by measuring proton-deuteron elastic scattering, with the deuteron being detected in the STT.

The data were taken in Spring 2011 at a beam energy of $1.826 \mathrm{GeV}$ and the results will not be available before 2012. However, to illustrate what one might expect from such measurements, Fig. 3 presents the first analysis of about 5\% of the data [15], showing the momentum of a "spectator" proton in the STT detected in coincidence with a $K^{+}$identified in the tracking telescopes. Note the abrupt cut-off at $\approx 60 \mathrm{MeV} / c$, caused by the need for the proton to go through the first STT layer. There is also a steep fall of the count rate at high momenta. This is to be expected, on the basis of the deuteron wave function, but, since these data are not yet acceptance corrected, no detailed comparisons can be made. To ensure the validity of the spectator model, a cut will be placed on the highest acceptable momentum, perhaps at $140 \mathrm{MeV} / \mathrm{c}$. 


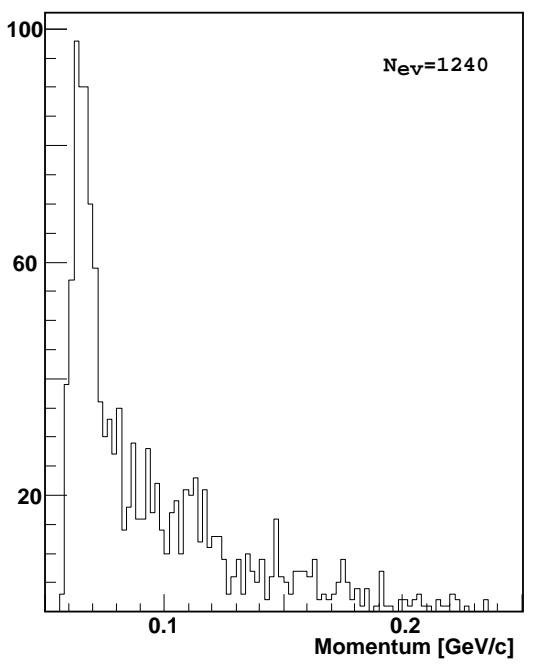

Figure 3: Laboratory momentum of a $K^{+}$from a $p d$ interaction at $1.826 \mathrm{GeV}$, detected in a range telescope at ANKE in coincidence with a slow proton in the STT.

The 1240 events in Fig. 3 suggest that the experiment might yield in total about 25k good $p d \rightarrow$ $p_{\mathrm{sp}} K^{+} n \Lambda$ candidates, spread over an excitation energy in the $K^{+} n \Lambda$ system from 0 to $120 \mathrm{MeV}$.

\section{Conclusions}

Hyperon production in proton-neutron collisions is very challenging experimentally. If one carried out inclusive $\mathrm{K}^{+}$production experiments on hydrogen and deuterium under identical conditions, one could achieve precisions on the cross section ratio of perhaps better than $10 \%$. The major uncertainty would be associated with the determination of the relative luminosity. The present ANKE data could therefore be improved, but at the cost of several weeks of beam time.

Using the COSY-TOF facility, one can measure many hyperon production channels exclusively with very high acceptance. In the $p d \rightarrow p_{\mathrm{sp}} K^{0} p \Lambda$ case, the identification of the tracks with particular decays is tricky but it is hard to envisage anybody else measuring large numbers of $K^{0}$. It is also not easy to work close to threshold due to the resulting small cross sections and hence higher relative backgrounds. Clearly we must wait for results of this first experiment.

COSY-ANKE has a very good $K^{+}$trigger but very small acceptance. Ideally, in the $p d \rightarrow$ $p_{\mathrm{sp}} K^{+} n \Lambda$ case one would like to see also the products of the $\Lambda \rightarrow p \pi^{-}$decay as well. This would then allow measurements to be carried out also above the $\Sigma$ thresholds. Unfortunately, the resulting count rates would make such a four-fold coincidence proposal hard to justify. Nevertheless, one should still get reliable cross sections closer to the $\Lambda$ threshold from the present experiment, but for these we must wait the full data analysis.

\section{Acknowledgments}

I am greatly indebted to Yury Valdau and Martin Krapp for providing me with extensive and very helpful information on the COSY-ANKE and COSY-TOF experiments, respectively. Support from the conference organisers is gratefully acknowledged. 


\section{References}

[1] Y. Valdau et al., Comparison of inclusive $K^{+}$production in proton-proton and proton-neutron collisions, arXiv:1109.0176 (nucl-ex).

[2] S. Abd El-Samad et al., Influence of $N^{*}$-resonances on hyperon production in the channel $p p \rightarrow K^{+} \Lambda p$ at 2.95, 3.20 and $3.30 \mathrm{GeV/c}$ beam momentum, Phys. Lett. B 688, 142 (2010).

[3] F. Balestra et al., Spin Transfer in Exclusive $\Lambda$ Production from $\vec{p} p$ Collisions at 3.67 GeV/c, Phys. Rev. Lett. 83, 1534 (1999).

[4] H. Calén et al., Measurement of the Quasifree $p+n \rightarrow d+\eta$ Reaction near Threshold, Phys. Rev. Lett. 79, 2642 (1997).

[5] G. Fäldt and C. Wilkin, Spin and Isospin Effects in the $N N \rightarrow N K \Lambda$ Reaction near Threshold, Eur. Phys. J. A 24, 431 (2005).

[6] E. Chiavassa et al., $\eta$-Meson Production in pd and pp Collisions, Phys. Lett. B 337, 192 (1994).

[7] R. I. Loutitt et al., Production of Strange Particles in p-p Collisions at 2.85 Bev, Phys. Rev. 123, 1465 (1961).

[8] M. Abdel-Bary et al., Production of $\Lambda$ and $\Sigma^{0}$ hyperons in proton-proton collisions, Eur. Phys. J. A 46, 27 (2010).

[9] M. Krapp et al., $\Lambda$ production in the reaction $p n(p) \rightarrow p K^{0} \Lambda(p)$ with deuterium target, IKP-Jülich Annual Report (2009); M. Krapp, Hyperon production in the reactions $p n(p) \rightarrow K^{0} \Lambda p(p)$ and $p p \rightarrow K^{+} \Lambda p$, contribution HK59.5 to the DPG meeting Münster (2011).

[10] M. Krapp, private communication.

[11] A. Baldini, V. Flamino, W. G. Moorhead, and D. R. O. Morison, Total Cross-Sections in High-Energy Reactions, in Landolt-Börnstein, New Series, Ed. H. Schopper (Springer-Verlag, Berlin, 1988).

[12] A. Dzyuba et al., Study of the energy dependence of the $p n \rightarrow K^{+} \Lambda n$ reaction close to threshold at ANKE, COSY proposal \#203 (2010).

[13] I. Lehmann et al., Spectator Detection for the Measurement of Proton-Neutron Interactions at ANKE, Nucl. Instrum. Methods Phys. Res. A 530, 275 (2004).

[14] H. J. Stein et al., Determination of target thickness and luminosity from beam energy losses, Phys. Rev. ST Accel. Beams, 11, 052801(2008).

[15] A. Dzyuba and Yu. Valdau, private communication. 